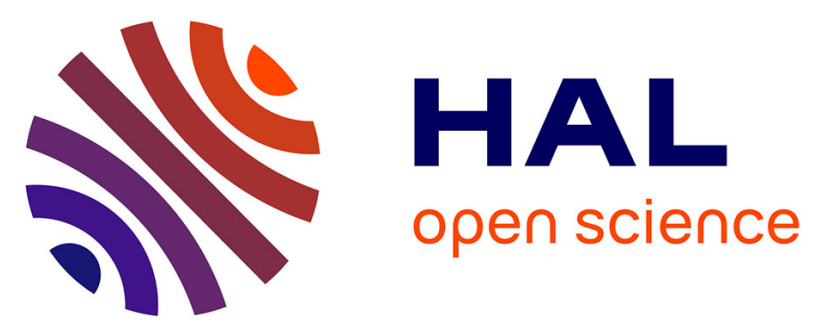

\title{
Analyse de la microstructure d'un horizon de surface enterré sous des matériaux archéologiques du Bronze ancien en Syrie (2200 BC)
}

\author{
Stéphane Gaffié, Ary Bruand, Marie-Agnès Courty
}

\section{To cite this version:}

Stéphane Gaffié, Ary Bruand, Marie-Agnès Courty. Analyse de la microstructure d'un horizon de surface enterré sous des matériaux archéologiques du Bronze ancien en Syrie (2200 BC). Comptes rendus de l'Académie des sciences. Série IIa, Sciences de la terre et des planètes, 2001, 332, pp.153160. 10.1016/S1251-8050(01)01517-8 . insu-00860784

\section{HAL Id: insu-00860784}

https://hal-insu.archives-ouvertes.fr/insu-00860784

Submitted on 11 Sep 2013

HAL is a multi-disciplinary open access archive for the deposit and dissemination of scientific research documents, whether they are published or not. The documents may come from teaching and research institutions in France or abroad, or from public or private research centers.
L'archive ouverte pluridisciplinaire HAL, est destinée au dépôt et à la diffusion de documents scientifiques de niveau recherche, publiés ou non, émanant des établissements d'enseignement et de recherche français ou étrangers, des laboratoires publics ou privés. 


\title{
Analyse de la microstructure d'un horizon de surface enterré sous des matériaux archéologiques du Bronze ancien en Syrie (2200 BC)
}

\author{
Stéphane Gaffié ${ }^{\mathrm{a}}$, Ary Bruand ${ }^{\mathrm{b}, \mathrm{c}, *}$, Marie-Agnès Courty ${ }^{\mathrm{d}}$ \\ a Laboratoire de science du sol, département «Agronomie-Environnement», Institut national agronomique Paris-Grignon, \\ 78850 Thiverval-Grignon, France \\ b Institut des sciences de la Terre d'Orléans, université d'Orléans, bâtiment de Géosciences, BP 6759, 45067 Orléans cedex 2, France \\ c Unité de science du sol, Inra, avenue de la Pomme-de-Pin, Ardon, BP 20619, 45166 Olivet cedex, France \\ d Laboratoire de science du sol, département «Agronomie-Environnement », CNRS-CRA, Institut national agronomique Paris-Grignon, \\ 78850 Thiverval-Grignon, France
}

Reçu le 16 octobre 2000 ; accepté le 8 janvier 2001

Présenté par Georges Pédro

\begin{abstract}
Microstructure analysis of a topsoil buried under archaeological materials from the Early Bronze Age in Syria (2200 BC). The microstructure of a buried topsoil in the Djezireh plain was compared with the microstructure of the present topsoil. The results from physicochemical analysis, the observations of the microstructure in scanning electron microscopy and the porosity measurements showed many similarities between the two soils. However the pore volume resulting from the aggregates packing and biological activity was smaller in the buried topsoil, but this difference might result from microstructure ageing after burying or due to a different microstructure prior to burying. In the latter the microstructure would result from surface conditions different from those that have determined the microstructure of the present topsoil. Finally, our observations showed the interest to measure more systematically physical parameters such as the porosity within surface deposits and soils that developed therein. (c) 2001 Académie des sciences / Éditions scientifiques et médicales Elsevier SAS
\end{abstract}

bulk density / scanning electron microscopy / palaeosol / organic matter

Résumé - La microstructure de l'horizon de surface d'un sol enterré dans les plaines de Djézireh a été comparée à celle de l'horizon de surface du sol actuel. Les résultats des analyses physico-chimiques, les observations effectuées en microscopie électronique et les mesures de porosité montrent de nombreuses similitudes entre les deux sols. Cependant, le volume de pores résultant de l'assemblage des agrégats et de l'activité biologique est plus faible dans l'horizon de surface du sol enterré. Cette différence pourrait être due, soit à un vieillissement de la microstructure postérieurement à l'enfouissement, soit à l'existence d'une microstructure différente avant l'enfouissement. Dans ce dernier cas, cette microstructure serait liée à des facteurs externes différents de ceux qui règnent à la surface du sol aujourd'hui. Enfin, nos résultats montrent l'intérêt qu'il y aurait à mesurer plus systématiquement des paramètres physiques tels que la porosité au sein des formations superficielles et des sols qui s'y sont différenciés. ๑ 2001 Académie des sciences / Éditions scientifiques et médicales Elsevier SAS

densité apparente / microscopie électronique à balayage / paléosol / matière organique

* Correspondance et tirés à part.

Adresse e-mail : AryBruand@orleans.inra.fr (A. Bruand). 


\section{Abridged version}

Many characteristics of the soil are related to climate, vegetation, faunal activity and human activities since the soil have been used by man. When a soil is buried under sediments, its characteristics can be preserved partly for a long time. Many studies have concerned the organic matter of buried topsoils and have showed that its content decreases with time after burying $[10,15,17,20]$. On the opposite, there is little information about the evolution of the structure after burying. Ruellan [13] and Mausbach et al. [9] indicated that structure would continue to evolve if the topsoil is buried under a small thickness of sediments because of the action of biological activity and dry/wetting cycles. When the thickness of sediments is greater than about $1 \mathrm{~m}$ the structure would be preserved, as attested by Courty [3] and Crowther et al. [4]. However, Crowther et al. [4] indicated that in such a case the soil structure can be affected by compaction resulting from overburden pressure. The objective of this study is to show that the microstructure of a topsoil has been preserved partially after burying which occurred several thousands of years ago. This potential memory of the soil after burying might be a source of information about the soil structure at the time of burying and more generally about the external factors that have determined that structure.

The study is part of the 'Tell Leilan Project' $[18,19]$. One of the main aims is the study of agriculture impact on soils and landscapes during the Early Bronze Age (3000-1900 BC). The study site is located in the Djezireh (Northeastern Syria) close to the tell of Abu Fara [12]. The soils developed on calcareous loamy-clay sediments and are calcic xerosols [5]. They have been cultivated since the Early Bronze Age. A soil buried under archaeological materials and located at $280 \mathrm{~m}$ of the northern limit of the tell Abu Fara was selected for study. A present soil located at $200 \mathrm{~m}$ north of the buried soil was selected for comparison. The soil morphology was described in the field and samples were collected for study in the laboratory. The physico-chemical characteristics were measured in the INRA's laboratory in Arras [7]. The specific volume of centimetric undisturbed but dried samples $\left(V\right.$, in $\mathrm{cm}^{3}$ per $\mathrm{g}$ of soil dried at $105{ }^{\circ} \mathrm{C}$ ) was measured using the kerosene method [11]. The specific volume of disturbed samples $\left(V_{\mathrm{r}}\right.$, in $\mathrm{cm}^{3}$ per $\mathrm{g}$ of soil dried at $105{ }^{\circ} \mathrm{C}$ ) prepared according to the method proposed by Fiès and Stengel [6] was also measured using the kerosene method. Other undisturbed samples were used for thin section preparation [8] and examination in scanning electron microscopy under the backscattered electrons mode [2].

In the field the buried soil and present soil showed many similarities (figure 1): a crumby structure in the topsoil which becomes progressively prismatic with depth, an increase in the secondary carbonates content with depth and the presence of ferruginous impregnation at the bottom. Laboratory analyses showed that the $\mathrm{CaCO}_{3}$ content was rather constant, except in the $0-30 \mathrm{~cm}$ and $30-60 \mathrm{~cm}$ of the present soil (table I). On the opposite the clay content of the non-calcareous fraction ranged from 30.6 to $44.0 \%$ and there was no relationship with depth (table I). The cation exchange capacity was about $22 \mathrm{cmol}_{\mathrm{c}} \cdot \mathrm{kg}^{-1}$ in the two soils. Reported to the noncalcareous clay fraction, the cation exchange capacity ranged from 55 to $73 \mathrm{cmol}_{\mathrm{c}} \cdot \mathrm{kg}^{-1}$ in the soil buried and from 54 to $72 \mathrm{cmol}_{\mathrm{c}} \cdot \mathrm{kg}^{-1}$ in the present soil. The higher exchangeable potassium content that was recorded in the soil buried under archaeological material would result from the presence of ashes in these materials. The organic carbon content decreased with depth in the two soils, being approximately twice as much at every depth in the present soil as in the buried soil (table I).

The topsoil of the present soil was characterised by small aggregates a few hundreds micrometres to one or two millimetres in diameter in a loose packing (figure $2 A$ ). Many polyconcave voids, resulting from this loose packing associated with much larger voids resulting from root and faunal activity, were observed. The topsoil of the buried soil under the archaeological materials showed also many small aggregates similar to those that were observed in the present soil but they were in a close packing (figure $2 B$ ). The pores resulting from that close packing were smaller than in the present soil but numerous large voids due to root and faunal activity were observed in the buried soil as well as in the present soil. The pore volume of disturbed samples can be considered as resulting from the close packing of elementary particles alone, i.e. without any contribution of pores resulting from either aggregate packing or biological activity. Thus assuming no variation of the particle density between samples, the volume of pores corresponding to the aggregates packing and biological pores $\left(V_{\mathrm{p}}\right.$, in $\mathrm{cm}^{3}$ per $\mathrm{g}$ of dried soil at $105^{\circ} \mathrm{C}$ ) was computed as follows:

$V_{\mathrm{p}}=V-V_{\mathrm{r}}$

Results show many similarities between the evolution of $V_{\mathrm{p}}$ with depth between the two soils, except in the first top centimetres (figure 3). These values of $V_{\mathrm{p}}$ were rather unexpected in the buried soil because the buried topsoil has been submitted to a pedostatic overburden pressure of about 350-400 $\mathrm{hPa}$. Indeed the buried soil was located in low topographical position where most of the soil is wetted during winter by top-down infiltration and capillary rise from the water table. Under these conditions the soil cohesion should be very low. Indeed for such a pressure and with wetted material, the porosity resulting from the aggregate packing might have been strongly affected by compaction as earlier shown by Sala and Tessier [14] and Bruand and Cousin [1] in the laboratory. Even for these unfavourable conditions, the microstructure specific to a topsoil and related porosity is still visible and measurable in the buried soil.

These results showed that the differences which were observed in scanning electron microscopy and with $V_{\mathrm{p}}$ between the two topsoils might result from ageing of structure after burying or from a microstructure present at the time of burying, thus indicating different external factors at that time. The presence of many biological pores in the buried 
S. Gaffié et al. / C. R. Acad. Sci. Paris, Sciences de la Terre et des planètes / Earth and Planetary Sciences 332 (2001) 153-160

topsoil and the close packing of aggregates are in favour of the second hypothesis thus indicating the presence of external factor leading to a close packing of aggregates without affecting the production of biological pores.

Finally, our results show that physical measurements such as porosity determinations should be performed more frequently and associated to the morphological description of surface deposits and soils as earlier proposed by Trichet and Courty [16]. The rather good conservation of the structure in the buried soil studied indicates that it might be still observable in other situations that are less favourable to memorising of soil characteristics.

\section{Introduction}

De nombreuses caractéristiques du sol sont en grande partie sous la dépendance de facteurs externes liés au climat, à la végétation, à la faune du sol et à l'homme, depuis que le sol est utilisé par ce dernier. Lorsqu'un sol est enfoui sous des matériaux naturels ou anthropiques, il est alors soustrait à l'action des facteurs externes; les caractéristiques du sol que ceux-ci déterminaient évoluent alors en fonction d'autres facteurs. Dans ce cadre général, les horizons de surface présentent un intérêt particulier. C'est en eux que des caractéristiques telles que la teneur en matière organique et sa composition sont étroitement liées aux caractéristiques de la végétation. C'est aussi dans ces horizons que la structure est la plus étroitement dépendante des conditions régnant à la surface $\mathrm{du}$ sol. En ce qui concerne la teneur en matière organique, de nombreux travaux ont montré que celleci diminue au cours du temps suite à l'enfouissement et à la minéralisation $[10,15,17,20]$. Cette décroissance de la teneur en matière organique peut être importante au point que les horizons de surface ne sont plus identifiables au sein des coupes [20]. En ce qui concerne la structure, il existe en revanche peu de données concernant son évolution suite à un enfouissement. Ruellan [13] et Mausbach et al. [9] indiquent que, dans le cas d'un enfouissement à faible profondeur (environ moins de $1 \mathrm{~m}$ ), la structure continuerait à évoluer sous l'effet de l'activité biologique et de cycles d'humectation-dessiccation saisonniers, au point que les propriétés acquises en surface ne seraient plus reconnaissables. En revanche, dans le cas d'un enfouissement plus profond, Courty [3] et Crowther et al. [4] indiquent que la structure de l'horizon de surface serait conservée partiellement après l'enfouissement. Crowther et al. [4] indiquent toutefois que la structure de l'horizon de surface pourrait disparaitre sous l'effet de la pression exercée par les couches de matériaux sus-jacentes.

L'objectif de cette note est de montrer que la microstructure d'un horizon de surface peut être en grande partie conservée pendant plusieurs milliers d'années à l'issue d'un enfouissement rapide. De tels résultats ouvrent des perspectives nouvelles quant à la connaissance de l'état structural des sols au moment de leur enfouissement et, plus généralement, quant à la connaissance des facteurs externes auxquels ils étaient soumis avant qu'ils ne soient enfouis. Ces résultats constituent aussi une référence pour discuter les conséquences de processus sédimentaires sur la pédogenèse.

\section{Présentation de l'étude}

Cette étude a été entreprise dans le cadre du programme international de recherches archéologiques Tell Leilan Project, dirigé par Harvey Weiss de l'université de Yale (États-Unis) [18, 19]. L'un des principaux objectifs de ce programme est l'étude de l'impact de l'agriculture sur l'évolution des sols et des paysages durant l'époque du Bronze ancien (30001900 BC). Le site d'étude est localisé dans les plaines de Djézireh (Nord-Est de la Syrie), à proximité immédiate du tell d'Abu Fara, situé actuellement dans une zone d'interfluve, donc hors d'apports sédimentaires alluviaux. Les tells, qui sont nombreux dans cette région, sont des monticules artificiels d'une centaine à plusieurs centaines de mètres de diamètre, formés par l'accumulation de matériaux de construction [12]. En Djézireh, ils datent pour la plupart de l'époque du Bronze ancien et sont autant d'anciennes agglomérations rurales ou urbaines, qui témoignent de l'ancienneté de l'anthropisation des paysages et, par conséquent, de la couverture de sol. Le climat actuel est de type méditerranéen semi-aride $(350 \mathrm{~mm}$ de précipitations annuelles). Les sols sont développés dans des matériaux argilo-limoneux calcaires, mis en place à différentes époques au cours de l'Holocène par apports alluviaux, puis éoliens. Les sols sont faiblement différenciés et sont pour la plupart des Calcic Xerosols [5]. Les seules différenciations morphologiques concernent le degré de développement de la structure, ainsi que le faciès et l'abondance des carbonates secondaires.

Les observations de terrain ont été réalisées durant l'automne 1995. Après une prospection de la zone d'extension du Tell d'Abu Fara, un profil de sol enterré et scellé sous environ $2 \mathrm{~m}$ de matériaux archéologiques a été mis à jour à $280 \mathrm{~m}$ au nord du Tell et choisi pour cette étude [7]. À $200 \mathrm{~m}$ au nord de ce premier profil, un profil de sol actuel a aussi été choisi pour référence. Après description de la structure sur le 


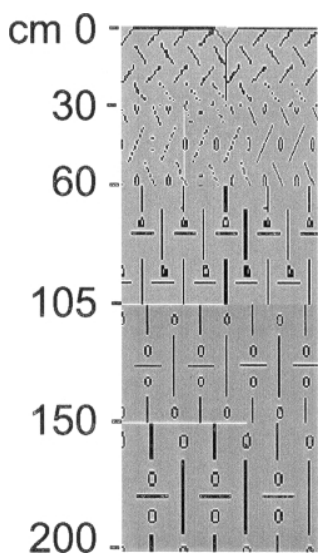

(A)

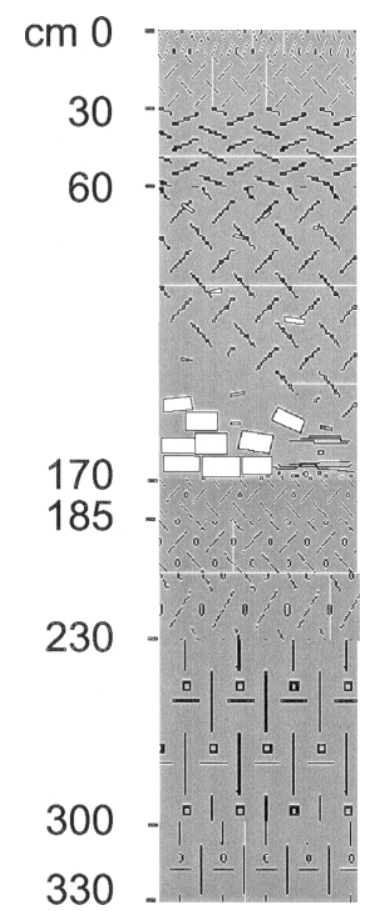

(B)
Figure 1. Profil de sol actuel et profil de sol enterré sous des matériaux archéologiques datés de l'âge du Bronze (2200 BC). Profil de sol actuel (A) : 0 à $30 \mathrm{~cm}$ : structure grumeleuse, fine et nette, quelques carbonates secondaires (pseudomycelium); 30 à $60 \mathrm{~cm}$ : structure polyédrique, fine et peu nette, carbonates secondaires (nodules pulvérulents); 60 à $105 \mathrm{~cm}$ : structure polyédrique à prismatique, grossière et peu nette, carbonates secondaires très nombreux (nodules); 105 à $150 \mathrm{~cm}$ : structure polyédrique à prismatique, grossière, peu nette, carbonates secondaires très nombreux (nodules); 150 à $200 \mathrm{~cm}$ : structure prismatique grossière et peu nette, très nombreux carbonates secondaires (nodules), imprégnations ferrugineuses. Profil de sol enterré (B) : 170 à $185 \mathrm{~cm}$ : structure polyédrique à grumeleuse, fine et nette, présence de quelques carbonates secondaires (nodules) et charbons de bois millimétriques; 185 à $205 \mathrm{~cm}$ : structure grumeleuse à polyédrique, fine et nette, présence de carbonates secondaires (nodules) et de quelques charbons de bois millimétriques; 205 à $230 \mathrm{~cm}$ : structure polyédrique à prismatique, fine et peu nette, présence de carbonates secondaires; 230 à $300 \mathrm{~cm}$ : structure polyédrique à prismatique, grossière et peu nette, présence de carbonates secondaires (nodules) et d'imprégnations ferro-manganiques; 300 à $330 \mathrm{~cm}$ : structure prismatique, grossière et peu nette, présence de carbonates secondaires (nodules) et d'imprégnations ferro-manganifères.

Figure 1. Present soil profile and soil profile buried under archaeological material of Bronze Age (2200 BC). Present soil profile (A): 0-30 cm: crumby structure, small and well developed, a few secondary carbonates (pseudomycelium); 30-60 cm: polyhedral structure, fine poorly developed, secondary carbonates (pulverulent nodules); 60-105 cm: polyhedral to prismatic structure, large and poorly developed, numerous secondary carbonates (nodules); $105-150 \mathrm{~cm}$ : polyhedral to prismatic structure, large and poorly developed, numerous secondary carbonates (nodules); $150-200 \mathrm{~cm}$ : large prismatic and poorly developed, numerous secondary carbonates (nodules), ferruginous impregnations. Soil profile buried (B): 170-185 cm: polyhedral to crumby structure, fine and well developed, a few secondary carbonates (nodules) and millimetric charcoal fragments; $185-205 \mathrm{~cm}$ : crumby to polyhedral structure, fine and well developed, a few secondary carbonates (nodules) and a few millimetric charcoal fragments; 205-230 cm: polyhedral to prismatic structure, fine and poorly developed, a few secondary carbonates (nodules); $230-300 \mathrm{~cm}$ : polyhedral to prismatic structure, large and poorly developed, numerous secondary carbonates (nodules) and ferromanganic impregnations; $300-330 \mathrm{~cm}$ : prismatic structure, large and poorly developed, numerous secondary carbonates (nodules) and ferro-manganic impregnations. terrain, des échantillons non perturbés ont été prélevés dans les principaux horizons (figure 1). Les échantillons ont été analysés par le laboratoire d'analyse des sols de l'Inra à Arras [7]. Le volume spécifique d'échantillons non fragmentés, de 3 à $6 \mathrm{~cm}^{3}$ de volume et séchés à l'air $\left(V\right.$, en $\mathrm{cm}^{3}$ par gramme de sol séché à $105^{\circ} \mathrm{C}$ ) a été mesuré à l'aide la méthode au pétrole [11]. Le volume spécifique d'échantillons remaniés $\left(V_{\mathrm{r}}\right.$, en $\mathrm{cm}^{3}$ par gramme de sol séché à $\left.105^{\circ} \mathrm{C}\right)$ préparé selon le protocole décrit par Fiès et Stengel [6] a aussi été mesuré, après séchage à l'air, à l'aide de la méthode au pétrole. D'autres échantillons non perturbés de dimensions décimétriques ont été prélevés pour la fabrication de lames minces [8]. La microstructure a été étudiée à faible grossissement en microscopie optique et à fort grossissement en microscopie électronique à balayage en mode électrons rétrodiffusés [2].

\section{Résultats et discussion}

À l'échelle du terrain, le profil enterré sous les sédiments archéologiques et le profil actuel présentent la même morphologie générale (figure 1). Ils sont tous deux caractérisés par une variation verticale de la structure : grumeleuse et de taille fine et nette dans les premiers décimètres, devenant plus grossière et passant progressivement en profondeur à une structure polyédrique de taille moyenne, puis à une structure prismatique de taille fine à moyenne. L'abondance des carbonates secondaires et des imprégnations ferrugineuses augmente aussi progressivement avec la profondeur. La teinte plus foncée et la structure grumeleuse de taille fine mise en évidence dans les trente premiers centimètres du profil de sol enterré indiquent la présence d'un horizon de surface dont l'organisation initiale aurait été préservée depuis l'enfouissement sous les matériaux archéologiques. La mise 
Tableau I. Caractéristiques physico-chimiques du profil de sol actuel et du profil de sol enterré sous des matériaux archéologiques du Bronze

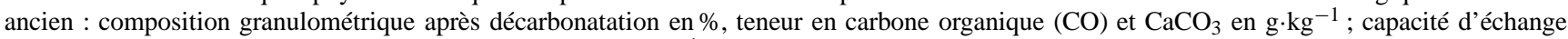
cationique (CEC) et teneur en cation échangeable en $\mathrm{cmol}_{\mathrm{c}} \cdot \mathrm{kg}^{-1}$.

Table I. Physico-chemical analysis of the present soil profile and of the soil profile buried under archaeological materials from the Early Bronze Age: particle size distribution after decarbonatation in \%, organic carbon content $(\mathrm{CO})$ and $\mathrm{CaCO}_{3}$ in $\mathrm{g} \cdot \mathrm{kg}^{-1}$; cation exchange capacity $(\mathrm{CEC})$ and exchangeable cations in $\mathrm{cmol}_{\mathrm{c}} \cdot \mathrm{kg}^{-1}$.

\begin{tabular}{|c|c|c|c|c|c|c|c|c|c|c|c|c|c|}
\hline \multirow{2}{*}{$\begin{array}{l}\text { Profondeurs } \\
\text { (cm) }\end{array}$} & \multicolumn{5}{|c|}{ Granulométrie $(\mu \mathrm{m})$} & \multirow[t]{2}{*}{$\mathrm{CaCO}_{3}$} & \multirow[t]{2}{*}{$\mathrm{pH}$} & \multirow[t]{2}{*}{$\mathrm{CO}$} & \multicolumn{5}{|c|}{ Complexe d'échange } \\
\hline & $<2$ & $2-20$ & $20-50$ & $50-200$ & $200-2000$ & & & & CEC & $\mathrm{Ca}^{2+}$ & $\mathrm{Mg}^{2+}$ & $\mathrm{Na}^{+}$ & $\mathrm{K}^{+}$ \\
\hline \multicolumn{14}{|l|}{ Sol actuel } \\
\hline $0-30$ & 37,2 & 21,8 & 7,4 & 1,5 & 0,2 & 306 & 8,6 & 6,4 & 23,2 & 22,7 & 2,5 & $<0,1$ & 0,6 \\
\hline $30-60$ & 40,6 & 17,6 & 6,0 & 1,4 & 0,2 & 324 & 8,6 & 3,7 & 22,1 & 21,5 & 2,8 & $<0,1$ & 0,3 \\
\hline $60-105$ & 32,6 & 21,3 & 4,4 & 0,9 & 0,1 & 380 & 8,7 & 1,7 & 22,6 & 18,0 & 5,5 & $<0,1$ & 0,1 \\
\hline $105-150$ & 31,8 & 20,7 & 4,6 & 1,0 & 0,1 & 392 & 8,7 & 1,4 & 23,0 & 17,2 & 7,1 & 0,1 & 0,1 \\
\hline $150-200$ & 44,0 & 11,3 & 3,5 & 0,8 & 0,1 & 375 & 8,7 & 1,1 & 23,9 & 17,2 & 8,5 & 0,1 & 0,1 \\
\hline \multicolumn{14}{|c|}{ Sol enterré sous les matériaux archéologiques } \\
\hline $170-185$ & 34,9 & 21,1 & 5,7 & 1,4 & 0,3 & 344 & 8,6 & 2,3 & 19,1 & 13,7 & 2,6 & 0,6 & 4,4 \\
\hline $185-205$ & 32,1 & 21,3 & 4,4 & 0,9 & 0,2 & 384 & 8,6 & 1,7 & 20,1 & 14,2 & 3,2 & 0,6 & 3,8 \\
\hline $205-230$ & 30,6 & 24,3 & 4,5 & 0,8 & 0,2 & 374 & 8,5 & 1,4 & 20,4 & 15,6 & 4,0 & 0,7 & 3,5 \\
\hline $230-300$ & 40,7 & 16,8 & 3,8 & 1,0 & 0,2 & 348 & 8,5 & 1,2 & 22,7 & 16,3 & 5,0 & 0,8 & 3,2 \\
\hline $300-330$ & 33,5 & 21,4 & 3,7 & 0,9 & 0,2 & 381 & 8,5 & 1,0 & 24,3 & 17,6 & 6,3 & 1,0 & 2,5 \\
\hline
\end{tabular}

en place de ces derniers correspond à la construction d'un mur en briques de terre crue, qui a été datée à 2200 BC environ sur la base d'observations archéologiques (Weiss, communication personnelle).

\subsection{Composition des sols}

Les sols étudiés se caractérisent par une teneur en carbonate qui varie peu, de 344 à $392 \mathrm{~g} \cdot \mathrm{kg}^{-1}$, si l'on écarte les horizons $0-30 \mathrm{~cm}$ et $30-60 \mathrm{~cm}$ du sol actuel, pour lesquels elle est sensiblement inférieure ( $t a$ bleau I). On note aussi la très faible teneur en grains non carbonatés de la taille des sables $(<1,7 \%)$ et la faible teneur en particules de la taille des limons grossiers $(<7,4 \%)$ (tableau I). En revanche, la teneur en argile varie de 30,6 à 44,0\%, mais cette variation n'est pas ordonnée avec la profondeur dans les deux profils de sols étudiés. Ceci est vraisemblablement à mettre en relation avec l'hétérogénéité de composition des matériaux parentaux dans lesquels les sols se sont développés. La capacité d'échange cationique (CEC) est voisine de $22 \mathrm{cmol}_{\mathrm{c}} \cdot \mathrm{kg}^{-1}$ dans les deux sols. Rapportée à la quantité d'argile granulométrique non carbonatée, la CEC varie de 55 à $73 \mathrm{cmol}_{\mathrm{c}} \cdot \mathrm{kg}^{-1}$ dans le profil de sol enterré et de 54 à $72 \mathrm{cmol}_{\mathrm{c}} \cdot \mathrm{kg}^{-1}$ dans le profil de sol actuel. De telles valeurs de CEC sont cohérentes avec les analyses minéralogiques effectuées sur des sols du même site, qui montrent que les minéraux argileux sont essentiellement des minéraux de type palygorskite et smectite [7]. Le complexe adsorbant est saturé essentiellement par le calcium, dont l'importance relative diminue en fonction de la profondeur au profit du magnésium. Toutefois, pour le profil enterré, les teneurs en potassium échangeable sont sensiblement plus élevées que dans le profil de sol actuel. Ceci est vraisemblablement la conséquence de la présence de niveaux archéologiques riches en cendres dans les matériaux sus-jacents qui scellent le profil de sol enterré.

La teneur en carbone organique décroît avec la profondeur dans les deux profils, mais elle est à chaque profondeur deux à trois fois supérieure dans le sol actuel (tableau I). Les horizons situés à la base sont caractérisés par des valeurs comparables voisines de $1 \mathrm{~g} \cdot \mathrm{kg}^{-1}$. Dans l'hypothèse où la teneur initiale en carbone organique de l'horizon de surface du sol enterré était comparable à celle de l'horizon de surface actuel, ceci indiquerait une perte importante de carbone organique depuis l'enfouissement sous les matériaux archéologiques. Une telle diminution a déjà été observée par de nombreux auteurs [10, 15, 17, 20].

\subsection{Microstructure des sols}

L'horizon de surface du sol actuel est caractérisé par la présence d'agrégats de très petite taille (de quelques centaines de micromètres à 1 ou $2 \mathrm{~mm}$ de diamètre), associant des grains de la taille des limons (quartz et carbonates) à une phase fine, constituée par des grains de la taille des argiles (figure 2A). Ces agrégats présentent un assemblage lâche; il en résulte un grand nombre de pores poly-concaves, dont la taille varie de quelques dizaines de micromètres à $1 \mathrm{~mm}$. De nombreux pores créés par des racines ou la macrofaune du sol sont aussi présents. Ils présentent des sections de forme arrondie et leur 

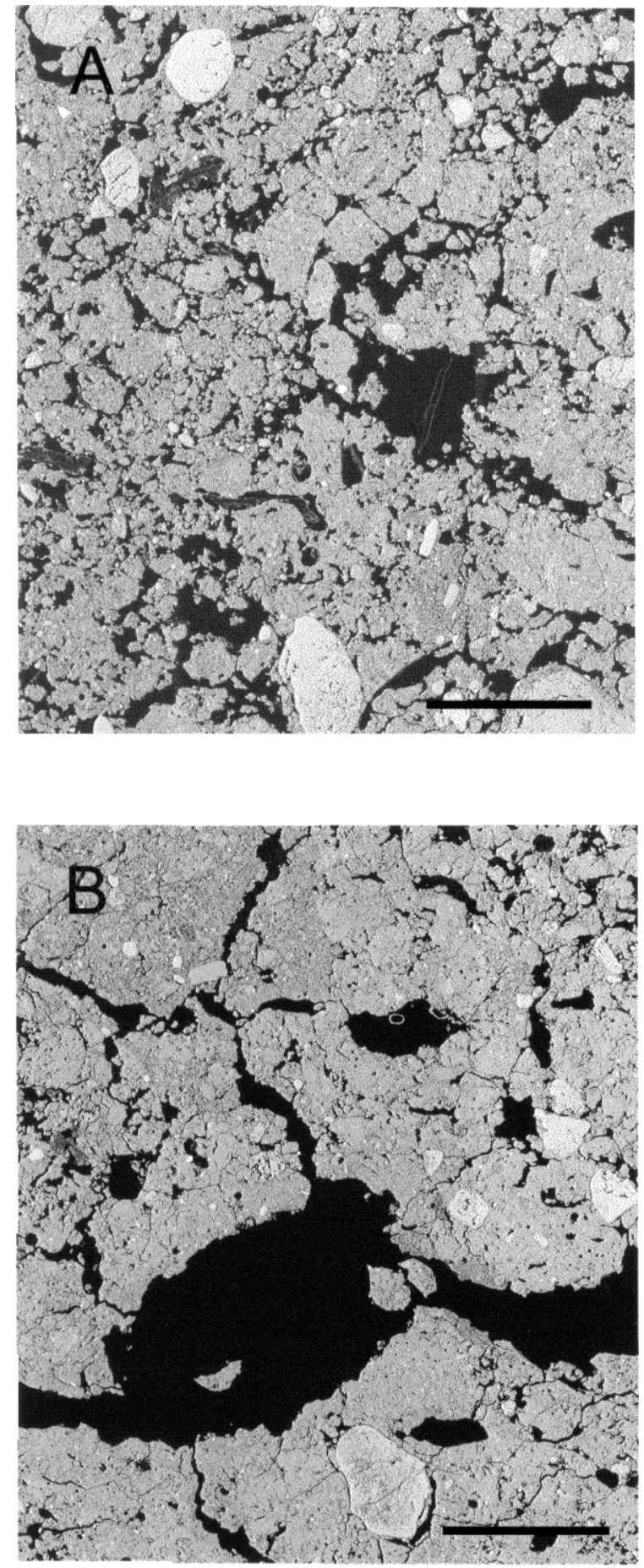

Figure 2. Observations effectuées en microscopie électronique à balayage en mode électrons rétrodiffusés : (A) horizon $0-30 \mathrm{~cm}$ du sol actuel, (B) horizon 170-185 cm du sol enterré. Les vides occupés par de la résine apparaissent en noir ; les plages gris clair correspondent aux grains de carbonates; les plages gris foncé correspondent aux grains de quartz et à la phase fine constituée de particules de la taille des argiles et des limons fins (échelle $=2 \mathrm{~mm}$ ).

Figure 2. Observations in scanning electron microscopy using backscattered electrons: (A) 0-30 cm horizon of the present soil, (B) 170 $185 \mathrm{~cm}$ horizon of the soil buried. Black areas are pores occupied by resin, light grey areas are carbonate, and dark grey areas correspond to quartz grains and clay particles (bar scale $=2 \mathrm{~mm}$ ). taille varie du millimètre à plusieurs millimètres. L'horizon de surface du sol scellé sous les matériaux archéologiques présente aussi de nombreux agrégats de taille équivalente à ceux décrits pour l'horizon de surface du sol actuel, mais ils sont beaucoup moins aisément discernables, en raison de leur assemblage compact (figure 2B). Les pores qui résultent de cet assemblage sont moins nombreux et de plus petite taille que dans l'horizon de surface du sol actuel. De nombreux pores de section arrondie, de taille millimétrique à pluri-millimétrique et liés à l'activité des racines et de la méso-faune du sol, sont aussi présents dans l'horizon de surface de ce sol enterré.

\section{3. Évolution du volume des pores après enfouissement}

Si l'on considère à présent les variations du volume spécifique des échantillons analysés, il apparaît une forte similitude entre les deux profils (tableau II). L'horizon de surface enterré présente des valeurs de volume spécifique élevées, comparables à celles mesurées dans l'horizon de surface du sol actuel. Le volume spécifique décroît ensuite régulièrement avec la profondeur pour les deux profils. Par ailleurs, l'horizon de surface du sol actuel et celui du sol enterré se caractérisent par une variabilité plus élevée du volume spécifique que dans les horizons sousjacents, ainsi qu'en témoignent les valeurs des écarts types sur la moyenne.

Compte tenu des faibles variations de composition entre les deux sols, on peut raisonnablement considérer que les variations de volume spécifique de la phase solide sont suffisamment faibles pour être négligées. Les variations du volume spécifique des échantillons sont alors le reflet des variations du volume de pores spécifique, c'est-à-dire du volume de pores par unité de masse de phase solide. Ce volume de pores peut être décomposé ( $i$ ) en un premier volume, qui correspond aux pores qui résultent de l'assemblage des particules élémentaires de la taille des argiles, limons et sables au sein des agrégats, et (ii) en un second volume, qui correspond aux pores qui résultent de l'assemblage des agrégats et de l'action des racines et de la méso-faune. Même si les variations de composition granulométrique sont faibles entre les différents horizons étudiés, elles sont suffisantes pour que les variations de volume de pores soient, au moins en partie, la conséquence de ces variations de composition. Puisque le volume spécifique des échantillons remaniés est la somme du volume spécifique de la phase solide - dont les variations sont considérées comme négligeables ici - et du volume de pores spécifique résultant de l'assemblage des particules élémentaires, tous les autres types de pores ayant été détruits lors du remaniement, nous avons calculé le volume spéci- 
Tableau II. Volume spécifique des échantillons non perturbés ( $V$, en $\mathrm{cm}^{3}$ par gramme de sol séché à $\left.105^{\circ} \mathrm{C}\right)$ et des échantillons remaniés $\left(V_{\mathrm{r}}\right.$, en $\mathrm{cm}^{3}$ par gramme de sol séché à $\left.105^{\circ} \mathrm{C}\right)$ dans le profil de sol actuel et dans celui du sol enterré.

Table II. Specific volume of the undisturbed samples $\left(V\right.$, in $\mathrm{cm}^{3}$ per gramme of oven-dried soil at $105{ }^{\circ} \mathrm{C}$ ) and specific volume of the reworked samples $\left(V_{\mathrm{r}}\right.$, in $\mathrm{cm}^{3}$ per gramme of oven-dried soil at $105{ }^{\circ} \mathrm{C}$ ).

\begin{tabular}{|c|c|c|c|c|}
\hline \multirow{3}{*}{$\begin{array}{l}\text { Profondeur } \\
\quad(\mathrm{cm})\end{array}$} & \multicolumn{4}{|c|}{ Volume spécifique } \\
\hline & \multicolumn{2}{|c|}{$\begin{array}{l}\text { des échantillons } \\
\text { non perturbés }\end{array}$} & \multicolumn{2}{|c|}{$\begin{array}{l}\text { des échantillons } \\
\text { remaniés }\end{array}$} \\
\hline & moyenne & écart type & moyenne & écart type \\
\hline \multicolumn{5}{|l|}{ Sol actuel } \\
\hline $0-30$ & 0,641 & 0,036 & 0,536 & 0,004 \\
\hline $30-60$ & 0,627 & 0,027 & 0,538 & 0,004 \\
\hline $60-105$ & 0,557 & 0,007 & 0,532 & 0,004 \\
\hline $105-150$ & 0,543 & 0,008 & 0,532 & 0,004 \\
\hline $150-200$ & 0,533 & 0,006 & 0,516 & 0,012 \\
\hline \multicolumn{5}{|c|}{ Sol enterré sous les matériaux archéologiques } \\
\hline $170-185$ & 0,635 & 0,007 & 0,574 & 0,014 \\
\hline $185-205$ & 0,613 & 0,021 & 0,544 & 0,007 \\
\hline $205-230$ & 0,564 & 0,011 & 0,532 & 0,004 \\
\hline $230-300$ & 0,538 & 0,011 & 0,524 & 0,002 \\
\hline $300-330$ & 0,544 & 0,006 & 0,520 & 0,002 \\
\hline
\end{tabular}

fique de pores résultant de l'assemblage des agrégats et de l'activité biologique $\left(V_{\mathrm{p}}\right.$, en $\mathrm{cm}^{3}$ par gramme de sol séché à $105^{\circ} \mathrm{C}$ ) dans les échantillons non perturbés de la façon suivante :

$V_{\mathrm{p}}=V-V_{\mathrm{r}}$

Un tel calcul repose sur l'hypothèse que le mode d'assemblage des grains élémentaires est, dans les échantillons remaniés, similaire à ce qu'il est au sein des échantillons non perturbés. Les valeurs de $V_{\mathrm{p}}$ ainsi calculées (figure 3) mettent en évidence une grande similitude de variation dans le profil de sol actuel et dans le profil de sol enterré.

Excepté dans les premiers centimètres de l'horizon de surface du sol enterré, où le volume poral a été vraisemblablement affecté lors de la construction du mur daté de l'âge du Bronze, les pores liés à l'assemblage des agrégats et à l'activité biologique sont encore largement présents. Les valeurs sont d'ailleurs voisines de celles mesurées dans le profil de sol actuel, alors que, depuis son enfouissement, on aurait pu s'attendre à une réduction plus importante du volume de pores. En effet, la zone où ont été étudiés les deux profils de sol est située en position basse dans la topographie et, par conséquent, est affectée par la remontée de la nappe en hiver. Même si la nappe ne se développe qu'à la base du profil de sol enterré, c'est l'ensemble du profil de sol enterré qui présenterait des teneurs en eau élevées lorsque la nappe est présente. Depuis son enfouissement, la surface du sol enterré serait ainsi

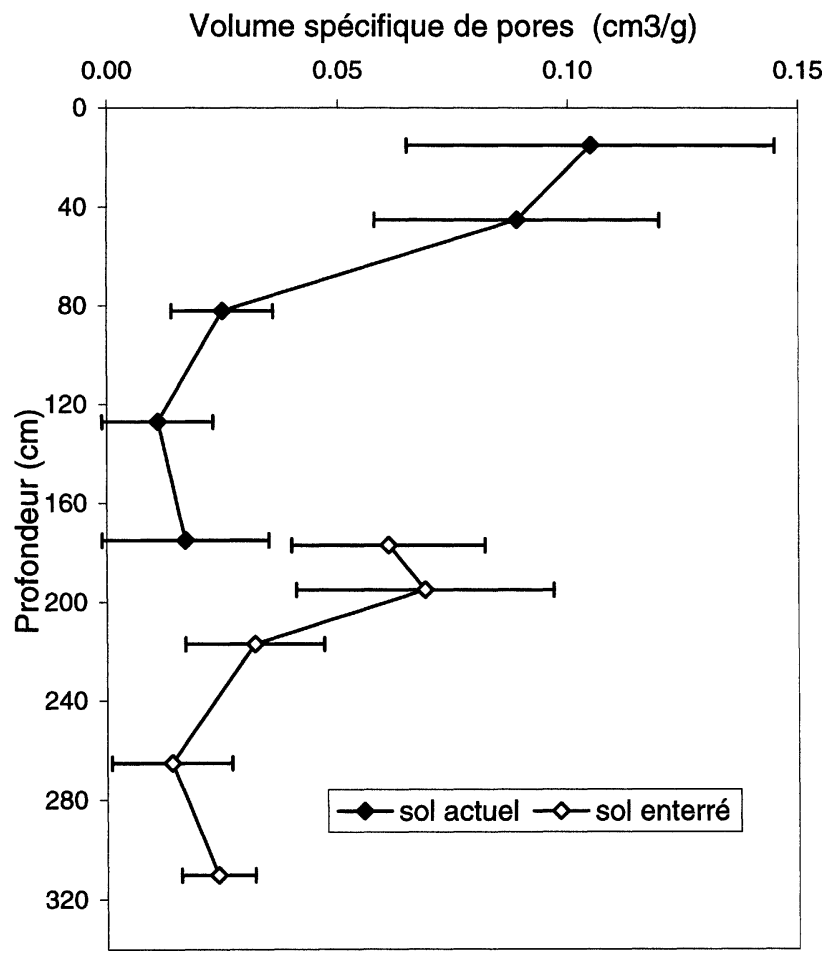

Figure 3. Volume spécifique de pores résultant de l'assemblage des agrégats et de l'activité biologique en fonction de la profondeur dans le profil de sol actuel et dans le profil de sol enterré.

Figure 3. Specific volume of the pores resulting from the aggregates packing and biological activity according to depth in the present soil and the soil buried.

soumise à une pression pédostatique de l'ordre de 350 à $400 \mathrm{hPa}$, lorsque le sol est humide. Même dans ces conditions de pression et d'humidité, pour lesquelles il a été montré expérimentalement que la porosité résultant de l'assemblage d'agrégats de quelques centaines de micromètres de diamètre [14] à quelques millimètres de diamètre [1] était largement affectée par le compactage, le volume de pores est encore aujourd'hui voisin de celui existant en surface dans le sol actuel. De plus, les observations effectuées en microscopie ont établi la présence de nombreux pores biologiques, alors que ceux-ci sont les plus aisément affectés lors d'un compactage.

\section{Conclusion}

Les résultats présentés dans cette note mettent en évidence le fait que la microstructure de l'horizon de surface du sol scellé par des matériaux archéologiques de l'âge du Bronze est encore reconnaissable et présente de nombreuses similitudes avec la microstructure de l'horizon de surface du sol actuel. Quant aux différences observées, elles peuvent résulter d'un vieillissement de la microstructure postérieur à l'en- 
S. Gaffié et al. / C. R. Acad. Sci. Paris, Sciences de la Terre et des planètes / Earth and Planetary Sciences 332 (2001) 153-160

fouissement ou témoigner de l'existence d'une microstructure plus compacte lorsque le sol était soumis aux facteurs externes antérieurement à son enfouissement.

Comme la teneur en carbone organique, la microstructure et la porosité qui lui est liée permettent d'identifier d'anciens horizons de surface au sein de coupes dans des formations superficielles. Cependant, si une teneur en carbone organique plus élevée, même légèrement plus élevée, se caractérise par une teinte plus foncée sur le terrain et peut, par conséquent, être aisément identifiée à cette échelle, l'identification d'une microstructure d'horizon de surface est en revanche plus difficile à réaliser lors d'une prospection sur le terrain. Ceci étant, l'apport potentiel d'une mesure plus systématique de paramètres physiques associée aux mesures physico-chimiques et à des observations morphologiques comme celles réalisées par Trichet et Courty [16] permettrait de mieux connaitre la nature des états physiques des sols dans le passé et en

\section{Références}

[1] Bruand A., Cousin I., Variation of textural porosity of a clayloam soil during compaction, Eur. J. Soil Sci. 46 (1995) 377-385.

[2] Bruand A., Cousin I., Nicoullaud B., Duval O., Bégon J.-C., Backscattered electron scanning images of soil porosity for analysing soil compaction around roots, Soil Sci. Soc. Am. J. 60 (1996) 895901.

[3] Courty M.-A., Le cadre paléogéographique des occupations humaines dans le bassin du Haut-Khabour (Syrie du Nord-Est). Premiers résultats, Paléorient 20 (1) (1994) 21-59.

[4] Crowther J., Macphail R.I., Cruise G.M., Short-term, postburial change in a humic rendzina soil, Overton Down experimental earthwork, Wiltshire, England, Geoarchaeology: An International Journal 11 (1996) 95-117.

[5] FAO-Unesco, Legend of the Soil Map of the World, Paris, 1977.

[6] Fiès J.-C., Stengel P., Densité texturale de sols naturels. I. Méthode de mesure, Agronomie 1 (1981) 651-658.

[7] Gaffié S., Évolution des horizons de surface au cours de l'Holocène. Étude des sols de la région de Tell Leilan (Syrie), thèse, Institut national agronomique de Paris-Grignon, Paris, 2000.

[8] Guilloré P., Méthode de fabrication mécanique et en série de lames minces. Doc. ronéotypé, Institut national agronomique de Paris-Grignon, département des Sols, Paris, 1985.

[9] Mausbach M.J., Wingard J.R., Gamble E.E., Modification of buried soil by postburial pedogenesis, Southern Indiana, Soil Sci. Soc. Am. J. 46 (1982) 364-369.

[10] Holliday V.T., Early and Middle Holocene soils at the Lubbock Lake archeological site, Texas, Catena 12 (1) (1985) 61-78.

[11] Monnier G., Stengel P., Fiès J.-C., Une méthode de mesure de la densité apparente de petits agglomérats terreux. Application à particulier lorsqu'ils étaient utilisés dans les contextes de la néolithisation des paysages et de leurs couvertures de sol.

Enfin, les microstructures observées dans l'horizon de surface du sol enterré sous des matériaux archéologiques de l'âge du Bronze nous conduisent aussi à nous interroger sur les possibilités de conservation de telles caractéristiques lors de conditions d'enfouissement moins rapides, comme c'est en particulier souvent le cas lorsqu'il s'agit d'un enfouissement par des matériaux naturels (colluvions, sédiments éoliens, sédiments fluviatiles...). Alors que l'on pouvait s'attendre à sa dégradation, compte tenu des conditions d'enfouissement et d'évolution au cours du temps, la relative bonne conservation de la microstructure dans le profil de sol enterré étudié permet de penser qu'elle pourrait aussi être conservée dans des conditions d'enfouissement et d'évolution moins favorables.

l'analyse des systèmes de porosité du sol, Ann. Agron. 24 (1973) 533-545.

[12] Rosen A.M., Cities of clays. The geoarcheology of tells, in: Butzer K.W., Freeman L.G. (Eds.), Prehistory Archeology and Ecology series, The University of Chicago Press, 1986, p. 167.

[13] Ruellan A., The history of soils: some problems of definition and interpretation, in: Yaalon D.H. (Ed.), Paleopedology: Origin, Nature and Dating of Paleosols, Israël University Press, Jerusalem, 1971, pp. 3-13.

[14] Sala G.H., Tessier D., Importance de l'état énergétique de l'eau sur l'aptitude au tassement de matériaux argileux non saturés, C. R. Acad. Sci. Paris, série II 316 (1993) 231-236.

[15] Schaetzl R.J., Sorenson C.J., The concept of 'buried' versus 'isolated' paleosols: examples from northeastern Kansas, Soil Sci. 143 (1987) 426-435.

[16] Trichet J., Courty M.A., Application de la micromorphologie des sols à la mise en évidence de pratiques d'irrigation dans la plaine d'Ai-Khanoum au cours de son occupation depuis l'âge du Bronze jusqu'à la période post-islamique, in: L'Asie centrale et ses rapports avec les civilisations orientales, des origines à l'âge du Fer, Mémoires de la Mission archéologique française en Asie centrale, tome I, Diffusion De Boccard, Paris, 1988, pp. 237-241.

[17] Watanabe M., Aoki K., Sakagami K., Humus accumulation in Holocene paleosols formed in Japanese tephra, Catena 34 (1998) $35-52$.

[18] Weiss H., Akkermans P., Stein G.J., Parayre D., Whiting R., Excavation at Tell Leilan, Syria, Am. J. Archeol. 94 (1990) 529-581.

[19] Weiss H., Courty M.A., Wetterstrom W., Guichard F., Senior L., Meadow R., Curnow A., The genesis and collapse of Third Millenium North Mesopotamian Civilization, Science 261 (1993) 995-1004

[20] Yaalon D.H., Soil-forming processes in time and space, in: Yaalon D.H. (Ed.), Paleopedology: Origin, Nature and Dating of Paleosols, Israël University Press, Jerusalem, 1971, pp. 29-39. 